\title{
Research on Low Frequency Amplifier Circuit for Small Signal
}

\author{
Fan Xuechao \\ Department of Electrical and Electronic Engineering, Suzhou College of Information Technology, \\ Suzhou, Jiangsu, China, 215200 \\ 346591653@163.com
}

Keywords: amplifier circuit, low frequency, small signal

\begin{abstract}
The amplifying circuit is designed to amplify small signal and realize the low noise amplification. This paper mainly studies and designs the small signal amplification circuit to make the weak signal to be amplified and transmitted without distortion. The research mainly includes the concept of low frequency amplifier circuit, the principle of circuit design, the design of suppressing noise and the debugging of the circuit.
\end{abstract}

\section{Introduction}

The output signal in the circuit detected by the sensor is often weak, among which some are in the millivolt level, such as the human body surface electrocardiograph in the microvolt, pyrometer output voltage. These signals are too small. How to amplify these small signals to several hundred millivolts even the volt level to some digital instrument display. The amplifying circuit needs to solve the problem of electronic technology in the "amplification" has two meanings: one is the value can be weak signal amplification to the people need to measure and use people; two is the requirement of signal waveform cannot be enlarged after the distortion. Some electronic systems require a greater power output, if the home audio system often need to the audio signal power increased to wattage or tens of watts. For general power larger signal amplification, the input signal amplitude is relatively high, in the process of amplification, even by noise interference. The output signal will not produce distortion. The weak signal output device similar to the sensor. It is related to the amplification and analysis of micro signal. Because the signal is weak, it is easy to be contaminated by noise. The noise is mainly caused by the noise of the environment noise, the noise of the circuit components itself. There is some other noise, such as circuit layout is not reasonable, electromagnetic compatibility, interference between the wires, etc. The noise runs penetrates the amplifying circuit and the effect become great. The normal signals are confused. Then the amplification circuit performance decline, the extracted signal impure and lower precision. So noise suppression is very important in small signal amplifier circuit.

The discrete component design of small signal low noise amplifier circuit not only can amplify the tiny signal to the signal range A/D conversion can accept, but also suppress the noise to a low level.

\section{Principle of Circuit Design}

The amplifier circuit can be divided into four types: a voltage amplifier circuit, a current amplifier circuit, a trans-impedance amplifier and a trans-conductance amplifier circuit. The basic process of amplifier includes the preamplifier, secondary amplifier, signal output. Finally, the amplifier circuit outputs the signals A/D conversion can accept. The process is as follows: 


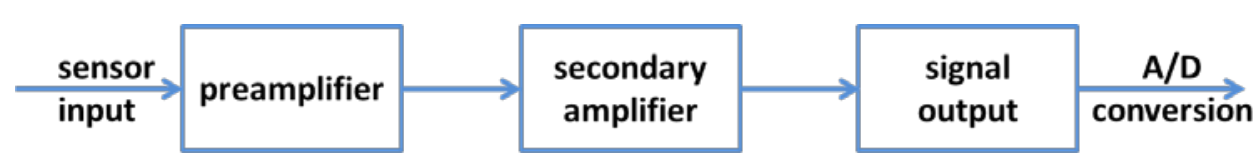

Fig. 1 Process of Amplified Circuit

Discrete circuits and integrated circuits have their own advantages and disadvantages. Discrete circuit is composed of a single component, such as transistors, diodes, resistors, capacitors, etc., which can be based on the intended target. It is designed to facilitate each parameter through debugging. You can change the parameters at any time. If you select the appropriate parameters, and components are high performance, through the design and debugging to change the three processes, so that the performance of the circuit to achieve the best. But although you can debug changes, if one of the aspects of the problem, the performance will be lower than the general integration of power amplifier. And integrated circuit for all the components are integrated in a silicon chip, small volume and the internal circuit using direct coupling together, especially integrated device parameters are stable, there is no need to adjust, signal to noise is relatively small. But of all the components of the integrated circuit integrated on a silicon chip, between tends to affect, if the a element by the external interference, the whole system will disorders, and design flexibility than discrete components, indicators, although good, but not high for general amplifier circuit, in the special and special high performance occasions, discrete circuit application more high, such as high performance headphone, high performance audio and so on. So it is very necessary to study the performance of high performance and integrated circuit of discrete components. Due to technical reasons, the base resistance of the integrated transistor is much larger than that of the discrete element, and the low frequency noise is larger than that of the discrete element. Also in the integrated circuit and reported the discovery of the plasma noise, and circuit structure similar to that of the integrated circuit noise in discrete components high. The design of the discrete can not only detects the signal, but also effectively inhibition the noise.

\section{Design of Suppressing Noise}

As the measured signal is very weak, we design the amplified circuit with discrete element through three stages, so that the amplified output signal can be used for data acquisition (A/D) and processing. However, the noise of the front stage is also amplified and the amplified ratio $\mathrm{F}$ is:

$$
F=F_{1}+\frac{F_{2}-1}{G_{2}}+\frac{F_{3}-1}{G_{1} G_{2}}+\mathrm{K}+\frac{F_{n-1}}{G_{1} G_{2} G_{3} \mathrm{~L} G_{n-1}}
$$

Among them, $G_{n}$ and $F_{n}$ are the effective power gain for the noise figure of the $N$ level. This shows that when the current set of gain $G_{1}$ is certain, and the noise figure $F_{1}$ enough, the noise ratio of the multi-stage series amplifier $\mathrm{F}$ will be smaller. So in the cascade amplifier preamplifier stage noise should be as small as possible. Due to the input stage, the differential amplifier circuit is used to suppress noise, and the voltage gain is small, and the amplification of the noise signal can be effectively suppressed. The amplifying circuit of preamplifier can get low noise characteristics of the input stage is by two identical triode tube composition difference divided into pre amplification circuit, the amplifier with high common mode rejection ratio, high input impedance, in order to achieve low noise. In the circuit, the positive and negative bias voltage is adopted, so the input end of the Q1 and the Q2 save the coupling capacitance and the bias resistance of the voltage divider are constructed. This preamplifier circuit and noise characteristics are not due to bias and great changes, so you can determine the reduced value of the distortion. Collector resistance RC and the level of the voltage gain, when need larger gain is open, increase collector resistance or increase of the 
active load circuit to enlarge the middle level, the complementary of tube composed of common emitter amplifier circuit was designed, the reasonable selection of static working point to reduce noise and to the output stage provides the noise of the input signal. Because the voltage gain of the entire circuit is basically determined by the circuit level, so as to increase the gain can be an appropriate increase in the collector resistance output stage is push-pull emitter follower, even under the condition of low load impedance with driving ability, and can reduce the waveform distortion of the whole circuit uses the voltage series negative feedback, between the input and output with a resistor form a feedback loop, the addition of negative feedback, increase feedback ring on signal to noise. Due to the nonlinear characteristic of the transistor, the output signal may be distorted. The negative feedback circuit, the signal output part and the input signal subtraction can reduce this distortion and improve the gain sensitivity.

\section{Analysis of the Debugging of the Circuit}

\section{A: Debugging of Quiescent Operation Point}

When the plus or minus 5 voltage is applied in the discrete amplification circuit, the voltage value of each node can be measured. The triode different voltage and current range in a different state make the collector current too small. The triode to cutoff region is too large. And the triode to the saturated zone is unable to normal amplification. To make transistor in the zoomed area, must ensure the triode emitter junction is forward biased, set electrical junction reverse biased. By measuring the voltage between the three poles of the transistor to ensure that the transistor emitter junction is biased. The collector junctions reverse bias is consistent with the principle of the design of the static operating point. The following is the input signal, a direct current power supply is switched on, with million with meter is used to measure the triode transistor amplifier quiescent point test data transistor in amplification, $\mathrm{U}_{\mathrm{BE}}$ measurement is about $0.6 \mathrm{~V}$, satisfying the NPN type triode tube emitter junction unbiased conditions. At the same time, through debugging, triode is closer to the off state. The triode is close to the saturated state. When the $\mathrm{I}_{\mathrm{CQ}}$ value is too low, triode tube will be closed. When the $\mathrm{I}_{\mathrm{CQ}}$ value is too high, triode tube will be saturated. Only the right set the quiescent operating point, amplifier can work normally to output the right current and low noise.

\section{B: Dynamic Voltage Output Debugging}

There is a large deviation between the voltage amplification ratio measured in the discrete amplification circuit and theory amplifying ratio. The theoretical calculation is the 99 times, and the highest actual test results magnification is only about 90 times, mostly in 87 times. These are mainly due to the discrete circuit resistor and a triode tube element more. These components existing parameter error and precision, from amplification multiples can be seen, discrete circuit under the same input signal, the magnification is stable, the output value fluctuation is very small, and so the stability of the circuit is good. From the frequency point of view, the discrete circuit at low frequency, the amplification factor is small, and to the higher frequency of $20 \mathrm{~K}$, the magnification is also increased to close to the theoretical value. Obtained by the integrated amplifying circuit of the measured results, the circuit voltage amplification multiples in the audio range are in 150 times fluctuates, and theory is very close, and in the range of low frequency $20 \mathrm{~Hz}-2 \mathrm{KHz}$, voltage amplification ratio of high, even up to 160 times. And by the high frequency of $20 \mathrm{kHz}$, the circuit voltage magnification declines to about 140 times. This is consistent with the response frequency of the actual operational amplifier chip. Discrete circuit measured value and the theoretical value of the difference is relatively large, and integrated circuit measured amplification

multiples and theoretical calculated values are very close, so the accuracy of the integrated circuit is relatively high, mainly due to the discrete circuit used in the many components, higher parameter 
error, and integrated circuit integrated in a block, caused by the parameter error. Discrete circuit measured value is stable, less volatile, and integrated circuit despite the high accuracy, but waves of ups and downs is bigger. The reason may is discrete circuit debugging. Static working point is relatively stable and integrated circuit due to the integrated chip in internal structure, only through the peripheral resistance of the rational allocation to adjust magnification, without taking into account the integrated chip of various internal compensation functions. The stability is relatively poor. When the discrete circuit is at low frequency, the amplification factor is smaller, and the theoretical value deviation is bigger. But when the higher frequency is $20 \mathrm{~K}$, the amplification factor also increases to be close to the theoretical value.

\section{C: Noise Indicators Debugging}

Generally speaking, the high precision digital all have real RMS function. The digital universal meter is used to calculate the input voltage to calculate the RMS value. RMS meter can be measured by a variety of different waveforms, but cannot be used for broadband noise measurement and spectrum analyzer to display the relationship between power and frequency, the and noise spectral density curve is similar, but belong to high precision instruments, so application is not very extensive. Oscilloscope to measure noise in the test, and the oscilloscope is divided into analog oscilloscope and digital oscilloscope, oscilloscope test on noise figure two as shown below, the analog oscilloscope, there are two limitations, one is unable to detect the low frequency noise, the other is the noise signal can trigger analog oscilloscope. Only repetitive waveforms can trigger the analog oscilloscope, when there is noise source input, analog oscilloscope shows a unique image, and according to the chapter on the analysis of noise, low frequency noise in the circuit is not only existent but it's very important in noise. The digital oscilloscope without the above limitations not only can detect the low frequency noise, but also can calculate the mathematical value of RMS.

\section{References}

[1] Liu Dayan, ZHU Huachen, Bai Ru, Qian Zhenghong, An Amplifier Circuit for Low Frequency and Small Signal Detection, J., Microelectronics, 2014, 44 (6): 746-749.

[2] Yin Zhuling, Xu Liangjun, The Analysis of Noise in Small Signal Amplifying Circuit, J., Electromechanical Components, 2011, 31 (6): 18-21.

[3] Dayan Liu, Design of an amplification circuit suitable for low-frequency and small-amplitude signal, D., Hangzhou Dianzi University, 2013.

[4] Yin Zhuling, Research on Low-Frequency Amplifier for Small Signal, D., Beijing University of Posts and Telecommunications, 2012. 